\title{
Erratum to: Carbon nanostructure morphology templates nanocomposites for phosphoproteomics
}

Susy Piovesana ${ }^{1}$, Daniel Iglesias ${ }^{2}$, Manuel Melle-Franco ${ }^{3}$, Slavko Kralj ${ }^{4}$, Chiara Cavaliere ${ }^{1}$, Michele Melchionna $^{2}$, Aldo Laganà ${ }^{1,5}$, Anna L. Capriotti ${ }^{1}(\bowtie)$, and Silvia Marchesan ${ }^{2}(\bowtie)$

${ }^{1}$ Piazzale A. Moro 5, Dipartimento di Chimica, Sapienza Universita di Roma, Rome 00185, Italy

${ }^{2}$ Via L. Giorgieri 1, Dipartimento di Scienze Chimiche e Farmaceutiche, Universita di Trieste, Trieste 34127, Italy

${ }^{3}$ CICECO, University of Aveiro, Aveiro 3810-193, Portugal

${ }^{4}$ Jamova 39, Department for Materials Synthesis, Jožef Stefan Institute, Ljubljana 1000, Slovenia

${ }^{5}$ Via Monteroni Campus Ecotekne, CNR NANOTEC, University of Salento, Lecce 73100, Italy

(c) Tsinghua University Press and Springer-Verlag GmbH Germany, part of Springer Nature 2020

\section{Erratum to}

Nano Research 2020, 13(2): 380-388

https://doi.org/10.1007/s12274-020-2620-4

The fourth author's name was unfortunately misspelled on the first page and the first page of the ESM.

Instead of

Susy Piovesana ${ }^{1}$, Daniel Iglesias ${ }^{2}$, Manuel Melle-Franco $^{3}$, Slavo Kralj ${ }^{4}$, Chiara Cavaliere ${ }^{1}$, Michele Melchionna $^{2}$, Aldo Laganà ${ }^{1,5}$, Anna L. Capriotti ${ }^{1}(\bowtie)$, and Silvia Marchesan $^{2}(\bowtie)$

It should read

Susy Piovesana ${ }^{1}$, Daniel Iglesias ${ }^{2}$, Manuel Melle-Franco $^{3}$, Slavko Kralj ${ }^{4}$, Chiara Cavaliere ${ }^{1}$, Michele Melchionna $^{2}$, Aldo Laganà ${ }^{1,5}$, Anna L. Capriotti ${ }^{1}(\square)$, and Silvia Marchesan $^{2}(\bowtie)$ 